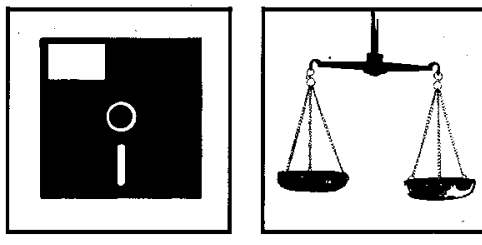

Urheberrechtsschutz für Computerprogramme

\section{Urheberrechtsschutz für Computerprogramme}

BGH, Urteilivom 4. Oktober 1990 - I ZR 139/89 - OLG Hamm, LG Bielefeld

\section{Leitsatz}

- Betriebssystem -

$\mathrm{Zu}$ den Anforderungen an die Darlegungslast des Klägers für die Urheberrechtsschutzfähigkeit eines Computerprogramms.

\section{Tenor}

Aufi die Revision der Klägerin wird das Urteil des 4. Zivilsenats des Oberlandesgerichts Hamm vom 27. April 1989 aufgehoben.

Die Sache wird zur anderweiten Verhandlung und Entscheidung, auch über die Kosten der Revision, an das Berufungsgericht zurückverwiesen.

\section{Tatbestand}

Der Streitpunkt. Die Klägerin stellt Datenverarbeitungsanlagen (Computer, Hardware) her und vertreibt diese; sie bietet für ihre Anlagen auch die dazugehörige Systemsoftware an. Die Beklagte zu 1 - eine $\mathrm{GmbH}$, die durch den Beklagten zu 2, ihren Geschäftsführer, vertreten wird - handelt mit gebrauchten Datenverarbeitungsanlagen der Klägerin, die sie von Dritten ankauft und (einschließlich der Systemsoftware) weiterveräußert und auch beim Erwerber installiert. Die Parteien streiten darüber, ob die Beklagte zu 1 die Systemsoftware der Klägerin im Rahmen der Weiterveräußerung in unzulässiger Weise benutzt und verwertet.

Vertragspolitik und $A G B$ 's der Die Klägerin schließt mit ihren Kunden über Hardware und Systemsoftware jeweils Klägerin gesonderte Verträge ab (sogenanntes Unbundling); und zwar über die Hardware entweder Miet-Leasing-oder Kaufverträge und über die Systemsoftware davon getrennte Lizenzverträge. In den Allgemeinen Geschäftsbedingungen (in der ab Februar 1984 geltenden Fassung) zu den Nutzungsüberlassungsverträgen der Klägerin heißt es unter Ziffi 4 zum Nutzungsumfang:

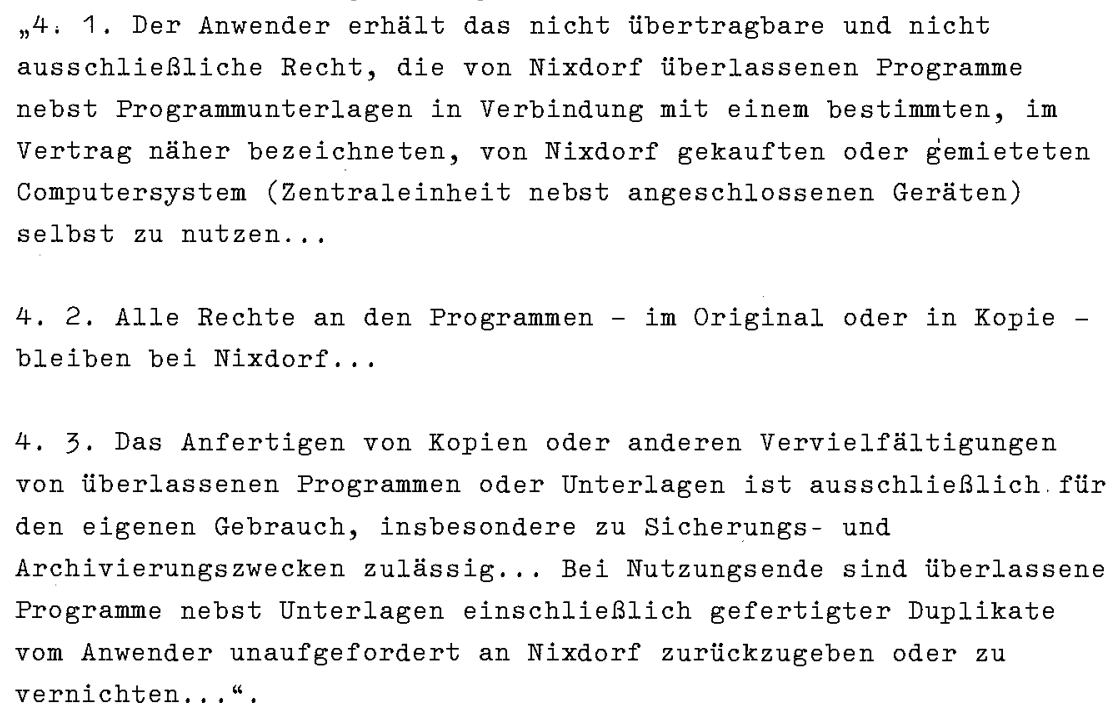

Das Streitzielder Klägerin

Die Rechtsmeinung der Klägerin

Die Klägerin nimmt die Beklagten wegen Urheberrechtsverletzung und Verstoßes gegen $\S 1$ UWG aufi Unterlassung, Auskunftserteilung sowie Feststellung der Schadensersatzpflicht in Anspruch.

Sie hat die Ansicht vertreten, ihre Systemsoftware sei urheberrechtlich geschützt. Durch die Weiterveräußerung der Computer nebst Systemsoftware werde ihr Verbreitungsrecht an den Programmen verletzt. Das von den Beklagten vorgenommene Einspeichern der Programme in den Arbeitsspeicher der Zentraleinheit stelle überdies eine umberechtigte Vervielfältigung dar. Außerdem griffen die Beklagten in den Fällen, in denen sie eine Speichererweiterung vornähmen, in ihre Betriebssysteme ein. 
Das Verhalten sei auch wettbewerbsrechtlich unter dem Gesichtspunkt des Leistungsschutzes und der Ausnutzung fremden Vertragsbruchs $z u$ beanstanden.

Die Klägerin hat zuletzt beantragt,

I. die Beklagten zu verurteilen,

1. es zu unterlassen, ohne Zustimmung der Klägerin Systemsoftware der Klägerin gemäß beigefügter Anlage A selbst zu nutzen, zu verwerten, zu verändern, durch Dritte verändern $\mathrm{zu}$ lassen, zu vervielfältigen, anzubieten, feil zuhalten, zu verkaufen oder sonst in den Verkehr zu bringen, sei es entgeltlich oder unentgeltlich, sei es in Verbindung mit Computersystemen der Klägerin oder mit anderen Computersystemen,

2. der Klägerin über den Umfang der vorstehend unter Ziffer I. 1. bezeichneten Handlungen Rechnung zu legen, und zwar jeweils insbesondere Auskunft über die Zahl der angebotenen oder verkauften Systemsoftware gemäß Anlage A, das Datum des Angebots oder Verkaufs, die Namen und Anschriften der Angebotsempfänger sowie der Käufer zu erteilen,

II. festzustellen, daß die Beklagten als Gesamtschuldner verpflichtet sind, der Klägerin allen Schaden zu ersetzen, der ihr aus den vorstehend unter Ziffer I. 1. bezeichneten Handlungen der Beklagten entstanden ist und künftig noch entstehend wird,

III.hilfsweise,

1. die Beklagten als Gesamtschuldner zu verurteilen, der Klägerin darüber Auskunft $\mathrm{zu}$ erteilen und Rechnung zu legen, welche Systemsoftware der Klägerin (Anlage A) sie an Kunden vermietet/lizenziert haben, und zwar unter Angabe des Datums des Vertragsabschlusses, der Anschrift des Kunden, der Dauer der Nutzung sowie der Höhe des monatlichen/jährlichen oder einmaligen Entgelts,

2. die Beklagten als Gesamtschuldner zu verurteilen, an die Klägerin einen nach Erteilung der Auskunft gemäß Ziffı 1 zu beziffernden Betrag nebst 4\% Zinsen zu zahlen.

Die Beklagten sind dem entgegengetreten. Sie haben die Urheberrechtsschutzfähigkeit der Systemsoftware der Klägerin in Abrede gestellt und sich im übrigen daraufiberufen, daß jedenfalls das Verbreitungsrecht nach $\S 17$ Abs. 2 UrhG erschöpft sei, weil die von der Klägerin vertriebene Zentraleinheit nebst dazugehöriger Systemsoftware ein einheitliches Ganzes darstelle. Die Programme würden von ihnen auch ohne eigene Vervielfäl- tigungsvorgänge weiterveräußert. Im Rahmen der Kapazitätserweiterungen würden sie nicht in unzulässiger Weise in das Betriebssystem der Klägerin eingreifen; denn es werde nur die Hardware geändert und diese Änderung dem Betriebssystem lediglich mitgeteilt. Das Landgericht hat die Klage nach Einholung eines Sachverständigengutachtens abgewiesen. Es hat zwar die Urheberrechtsschutzfähigkeit der Systemsoftware bejaht, ist jedoch von einer Erschöpfung des Verbreitungsrechts ausgegangen.

Die Berufung ist ohne Erfolg geblieben (OLG Hamm GRUR 1990, 185 ff).

Mit der Revision verfolgt die Klägerin ihre ursprünglichen Klageanträge weiter.

Die Beklagten beantragen, die Revision zurückzuweisen.

\section{Entscheidungsgründe}

I.

Das Berufungsgericht hat sowohl urheber- als auch wettbewerbsrechtliche Ansprüche verneint und dazu ausgeführt:

Die Urheberrechtsschutzfähigkeit der streitgegenständlichen Programme lasse sich nicht feststellen, da es insoweit an einem substantiierten Vorbringen der Klägerin fehle. Die Klägerin hätte im einzelnen darlegen und in körperlich wahrnehmbarer Form plastisch machen müssen, welche Leistungen, die in den Programmbestandteilen Niederschlag gefunden hätten, den erforderlichen Schöpfungsgrad erreichten. Ihr Vorbringen sei jedoch nur allgemein gehalten und lasse es nicht zu, den gebotenen Gesamtvergleich der in Frage stehenden Programme mit vorbekannten Gestaltungen vorzunehmen; es - fehle an einer der konkreten sinnlichen Wahrnehmung zugänglichen Synopse der konkreten und vorbekannten Formgestaltungen. Dem Vorbringen der Klägerin lasse sich auch nicht entnehmen, wo Auswahl, Sammlung, Anordnung und Einteilung der Informationen urheberrechtlich faßbaren Kategorien entsprächen. Die Darstellung beschränke sich auf die Umschreibung von Problemanalysen, die wiedergabetechnischen Eigenschaften der Anlage, die Schilderung von Arbeitsvorgängen, technischen Erweite-

Der Beklagtenvortrag

\section{Klageabweisung durch das Landgericht}

\section{Erfolglosigkeit der Berufung} Das Revisionsziel

\section{OLG Hamm:}

Weder Ansprüche aus Urheberrecht noch Ansprüche aus

Wettbewerbsrecht

Kein substantiierter. Vortrag.der Klägerin zur urheberrechtlichen Schutzfähigkeit der Programme 


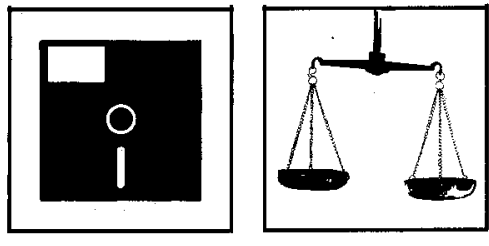

OLG Hamm:

Wegenfeblender. Substantiierung keine Einholung eines

Sachverständigengutachtens

Klagevorbringeñü die Annabme einer wettbewerblichen Eigenart nicht ausreichend:

OLG Hamm:

Kein wettbewerbsrechtlicher Leistungsschutz

Was verstand Nixdorf unter „Systemsoftware"?

Der Unterlassungsantrag ist nicht bestimmt!genug

Wie ,individualisiert" man Softzeare?
Urheberrechtsschutz für Computerprogramme

rungsmöglichkeiten u.ä.; für eigentümlich gehaltene Leistungen würden nur kursorisch umschrieben, ohne daß erkennbar sei, ob die gewählten Strukturen technisch bedingt seien oder auf gestalterischen Elementen beruhten. Außerdem werde im wesentlichen auf den Inhalt der gefundenen Probleme abgestellt. Als beispielhaft für die unzureichende Substantiierung seien die Ausführungen zu den Systemen „TACOS“, „DEGAS“, „NIROS“ einschließlich der Dienstprogramme „Sysmod“ und „Fernbetreuung" sowie zum Kapitel „Compiler“ zu nennen. Das Kapitel „Fimas, Sorbas, Diarep“ befasse sich zwar ausführlich mit der Problemanalyse und der gefundenen Problemlösung, mache aber formgestalterische Strukturen nicht sinnlich faßbar. Angesichts der fehlenden Substantiierung komme die Einholung eines Sachverständigengutachtens nicht in Betracht.

Die Klage sei weiter nicht unter dem Gesichtspunkt des ergänzenden wettbewerbsrechtlichen Leistungsschutzes begründet, da das Klagevorbringen für die Annahme einer wettbewerblichen Eigenart nicht ausreiche. Es brauche deshalb auch nicht geklärt zu werden, ob der Handel der Beklagten mit gebrauchten Geräten der Klägerin unter Verwendung, der Systemsoftware die Ausnutzung fremden Vertragsbruchs darstelle.

IL

Die Revision hat Erfolg. Sie führt zur Aufhebung und Zurückverweisung.

1. Streitgegenstand ist die als Anlage A zum Klageantrag aufgeführte Systemsoftware „jeweils in sämtlichen Programmversionen“ für fünf Computerbaureihen der Klägerin:

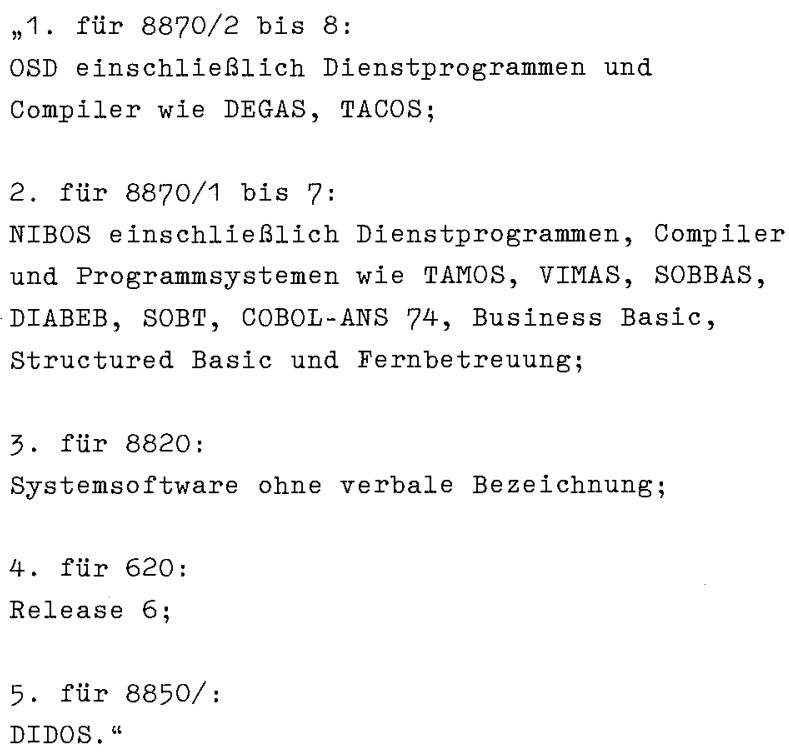

Die Klägerin versteht dabei nach ihrem Klagevorbringen unter Systemsoftware „Betriebssysteme, Übersetzungsprogramme, Programmgeneratoren, Dienstprogramme, Testhilfen und systemnahe Software" (vgl. auch Ziff: 1 der AGB der Klägerin für die Überlassung von Systemsoftware). Die Begriffsbestimmung in diesem Bereich ist allerdings nicht einheitlich. So wird (u. a.) teils zwischen Betriebssystemen, Systemsteuerprogrammen und Systemprogrammen unterschieden, wobei zum Betriebssystem auch Compiler, Dienstprogramme u. a. gerechnet werden; teils wird von systembezogener bzw. systemnaher Software oder von Systemsoftware als Oberbegriff für die Gesamtheit aller systembezogenen Programme gesprochen (vgl. Lichtenberg, CuR 1986, 521 f). Angesichts des Fehlens eines einheitlichen Begriffsverständnisses und des Umstandes, daß die in Anlage A zum Klageantrag aufgeführten Programmbezeichnungen eine nähere inhaltliche Bestimmung des Programmgegenstandes nicht erlauben, sind die vom Berufungsgericht hinsichtlich der Bestimmtheit des Unterlassungsantrags geäuBerten Bedenken berechtigt. Die Klägerin wird im wiedereröffneten Berufungsrechtszug Gelegenheit zu der im Blick auf die Vollstreckung gebotenen Individualisierung ihres Antrages haben; dies kann auf dem Wege einer näheren Beschreibung der Programme oder einer Bezugnahme auf Programmträger oder Programmausdrucke erfolgen (vgl. zum letzteren BGHZ 94, 276, 291 f-Inkasso-Programm; auch Schulze, CuR 1989, 800). 
2. Die streitgegenständliche Systemsoftware ist einem Urheberrechtsschutz für Datenverarbeitungsprogramme nach $\S 2$ Abs. $1 \mathrm{Nr}$. 1 UrhG grundsätzlich zugänglich. Die vom Berufungsgericht angedeuteten Bedenken, es käme möglicherweise nur ein Patentschutz in Betracht, sind unbegründet. Zum einen würde ein solcher Schutz einem Urheberrechtsschutz nicht zwingend entgegenstehen, zum anderen scheidet er hier aber auch aus. Nach $\S 1$ Abs. 2 Nr. 3, Abs. 3 PatG sind „Programme für Datenverarbeitungsanlagen" als" solche nicht als Erfindungen anzusehen. Damit sind alle Computerprogramme nicht technischer Natur vom Patentschutz ausgenommen. Dies gilt allerdings nicht für Programme technischer Natur (vgl. BGHZ 67, 22, 29 - Dispositionsprogramm; BGH, Urt. v. 13. 5. 1980 - X ZB 19/78, GRUR 1980, 849, 850 - Anti-Blockiersystem; Benkard/Bruchhausen, PatG, 8. Aufl. 1988, § 1 Rdn. 104 m.w. N.). Betriebssysteme der vorliegenden Art, die lediglich der Steuerung eines Computers und der mit ihm verbundenen Anschlußgeräte dienen (vgl. nachfolgend unter II. 3. b), stellen keine technischen Programme in diesem Sinne dar (vgl. BPatG CuR 1988, 652, 654 fs siehe auch BPatG GRUR 1987, 31, 32 - Elektronisches Übersetzungsgerät). Auch in der vom Berufungsgericht angeführten Entscheidung der technischen Beschwerdekammer des Europäischen Patentamts (EPA GRUR Int. 1987, 173, $175=$ CR 1986, 537, 539 f; dazu Benkard/Bruchhausen aaO, $\S 1 \mathrm{Rdn} .104)$, die eine computerbezogene Erfindung zum Gegenstand hatte, ging es, nicht um den Patentschutz für ein dem Betriebssystem vergleichbares Programm; der aufi ein technisches Verfahren gerichtete Patentanspruch wurde nicht als ein aufi ein Computerprogramm als solches bezogener Schutzanspruch angesehen.

3. Die Annahme des Berufungsgerichts, die Klägerin habe nicht substantiiert dargetan, daß die streitgegenständliche Systemsoftware eine für den Urheberrechtsschutz erforderliche persönliche geistige Schöpfung im Sinne des $§ 2$ Abs. 2 UrhG darstelle, hält der rechtlichen Nachprüfung nicht stand. Das Berufungsgericht hat zu hohe Anforderungen an die Darlegungslast der Klägerin gestellt.

a) Die dem Kläger im Urheberrechtsverletzungsprozeß obliegende Darlegungslast für das Vorliegen einer persönlichen geistigen Schöpfung erfordert grundsätzlich die konkrete Darlegung der die Urheberrechtsschutzfähigkeit begründenden Elemente (vgl. BGH, Urt. v. 10. 10. 1973 - I ZR 9 3/7 2, GRUR 1974, 740, 741 - Sessel). Die Frage, welche Anforderungen im Einzelfall zu stellen sind, hängt wesentlich von der konkreten Werkart ab. So sind z. B. bei Kunstwerken keine überhöhten Anforderungen an die Darlegungslast zu stellen. Denn bei ihnen ist die Schwierigkeit nicht zu verkennen, ästhetisch wirkende Formen überhaupt mit den Mitteln der Sprache auszudrücken (vgl. BGH GRUR 1974, 740, 741 - Sessel m.w. N.). Die für den ästhetischen Eindruck wesentlichen Formmerkmale einer schöpferischen Leistung entziehen sich oft der genauen Wiedergabe durch Worte, so daß eine erschöpfende Einzelaufgliederung der künstlerischen Elemente in der Regel nicht erwartet werden kann (vgl. BGHZ 5, 1, 4 - Hummelfiguren I). Wesentlich ist bei solchen Werken der sich aufgrund der Betrachtung des Objekts ergebende Gesamteindruck. Bei Computerprogrammen scheidet demgegenüber eine unmittelbare Anschauung der konkreten Formgestaltung durch den Richter grundsätzlich aus. Denn sie weisen die Besonderheit auf, daß das in einer höheren Programmiersprache (z. B. BASIC, FORTRAN, PASCAL, C) abgefaßte Quellenprogramm nicht allgemein, sondern nur für den Fachmann verständlich ist; das in einer dem Computer verständlichen Maschinensprache verfaßte Objektprogramm ist in der Regel ohnehin nicht unmittelbar lesbar. Der Richter ist danach aufi eine verständliche Programmbeschreibung, aus der sich die schöpferischen Elemente ergeben, angewiesen. Dabei ergibt sich für den darlegungspflichtigen Kläger die Schwierigkeit, die formgestalterischen Strukturen seines Programms, dessen Beurteilung vorliegend seiner Natur nach in gewissem Umfang sogar ein Verständnis der Technik der Hardware voraussetzt (vgl. nachfolgend unter II. 3. b), auch für den Nichtfachmann nachvollziehbar zu umschreiben. Das Fehlen eines unmittelbaren Anschauungsobjekts schließt es überdies nahezu aus, die formgestalterischen Strukturen in dem vom Berufungsgericht geforderten Sinne "sinnlich faßbar" zu -machen. Auf „theoretische Umschreibungen“ und „Abstraktionen“ wie sie vom Berufungsgericht beanstandet werden, kann nicht völlig verzichtet werden. Das Gericht ist gehalten, sich das notwendige Verständnis erforderlichenfalls mit Sachverständigenhilfe zu verschaffen.

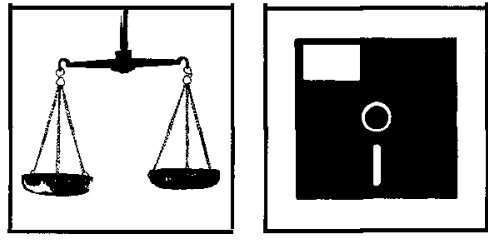

Patentschutz scheidet aus (anders wobldas OLG Hamm)

Das OL G Hamm stellte $\mathrm{z} u$ bohe Anforderungen an die Darlegungslast zum Merkmal „ppersönliche geistige Schöpfung.“

Darlegungslast im Urheberrechtsverletzungsprozeß

\section{Kunstzererk:}

keine überböbten Anforderungen

\section{Besonderheiten bei \\ Computerprogrammen}

Ein Anschauungsobjekt fehlt. 


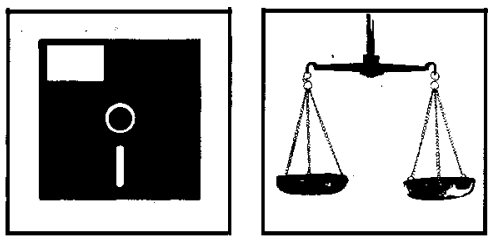

Im Streitfalligeht es um die identische Benutzung.von Software.

Hinreichende Gestaltungshöhe: Abstandivom Durchschnitt

Das Klagevorbringen erfüllt die Darlegungslast

Die Rolle des Betriebssystems nach Nixdorfi

Beispielerfür Gestaltungsmöglichkeiten beim Betriebssystem
Die Anforderungen an die Darlegungslast werden weiter dadurch bestimmt, ob der konkrete Fall eine genaue Festlegung der schöpferischen Elemente und damit des Schutzumfangs erfordert. Werden z. B. nur einzelne Arbeitsergebnisse der Vorstufen bzw. Teile des Programms übernommen oder besteht bei mehreren an der Programmerstellung Beteiligten Streit über den jeweils geleisteten Anteil, so wird es daraufiankommen, ob die konkret in Rede stehenden Teile schöpferische Eigentümlichkeit aufweisen. Bei der identischen Übernahme eines Computerprogramms (der 1:1-Kopie) wird eine ins einzelne gehende Herausarbeitung aller schöpferischen Elemente entbehrlich sein, sofern überhaupt eine schöpferische Eigentümlichkeit - an welcher Stelle des Programms oder seiner Entwicklungsstufen auch immer - festgestellt werden kann. So liegt es auch im Streitfall, in dem es - von den durch die Kapazitätserweiterungen bedingten Eingriffen abgesehen - um die identische Benutzung und Verwertung der Systemsoftware der Klägerin geht.

Im einzelnen bestimmt sich der Umfang der Darlegungslast im wesentlichen nach den allgemeinen urheberrechtlichen Grundsätzen, wobei es für den Urheberrechtsschutz von Computerprogrammen im allgemeinen aufi die Form und Art der Sammlung, Einteilung und Anordnung des Materials ankommt (vgl. BGHZ 94, 276, 285 - InkassoProgramm). Die Frage der schöpferischen Eigentümlichkeit bemißt sich nach dem Gesamtvergleich gegenüber vorbestehenden Gestaltungen. Lassen sich nach Maßgabe dieses Gesamtvergleichs schöpferische Eigenheiten feststellen, so sind diese dem durchschnittlichen Schaffen bei der Programmerstellung gegenüberzustellen. Eine für die Urheberrechtsschutzfähigkeit hinreichende Gestaltungshöhe wird erst erreicht, wenn das allägliche, durchschnittliche Programmiererschaffen, das auf einer mehr oder weniger routinemäßigen, handwerksmäßigen, mechanisch-technischen Aneinanderreihung und Zusammenfügung des Materials beruht, deutlich überstiegen wird.

b) Diesen Anforderungen an die Darlegungslast genügt das Klagevorbringen.

Die Klägerin hat zum besseren Verständnis der streitgegenständlichen Systemsoftware vorab ganz allgemein Bedeutung, Funktion und Struktur des Betriebsprogramms einer Datenverarbeitungsanlage dargelegt. Sie hat dabei herausgestellt, daß das Betriebssystem im Gegensatz zur Anwendersoftware nicht der Lösung eines individuellen betrieblichen Problems dient (wie z. B. das der Senatsentscheidung BGHZ 94, 276 ffi zugrundeliegende Inkasso-Programm), sondern die Funktion hat, die Steuerung des Computers und der mit ihm verbundenen Anschlußgeräte in Verbindung mit der Anwendersoftware zu übernehmen. Betriebsprogramme übernehmen als Bindeglied zwischen der Hardware und dem Benutzer u. a. die Steuerung der Ein- und Ausgabe von Daten, die Speicherverwaltung, die Übersetzung der in einer höheren Programmiersprache geschriebenen Anwenderprogramme in die Maschinensprache (Compiler), die erweiterte Unterstützung von Einheiten der Datenfernverarbeitung, die Steuerung der simultanen Arbeit mehrerer oder einer Vielzahl von Endbenutzern oder Dienstprogrammfunktionen (vgl. auch BPatG GRUR 1987, 31, 32).

aa) Die Klägerin hat dargelegt, daß der geistig-schöpferische Gehalt eines Betriebsprogramms seinen Niederschlag und Ausdruck insbesondere in der Form und Art der Sammlung, Auswahl und Gliederung der zur Steuerung des Computers und der mit ihm verbundenen Anschlußgeräte erforderlichen Befehle finde. Insoweit bestehe ein erheblicher Gestaltungsspielraum, der hier keineswegs durch die vorgegebene Hardware technisch eingeengt sei. Als Beispiele für unterschiedliche technische Gestaltungsmöglichkeiten bei den verschiedenen Systemen und den Umfang möglicher Funktionen hat die Klägerin u.a. angeführt: stapelverarbeitungsorientierte oder dialogorientierte Verarbeitung, Verwaltung von Plattenabschnitten im Hauptspeicher durch Anwendung wirtschaftlicher Methoden, Prüfsummenbescheinigungen mit unterschiedlichem, internem Zeitverhalten, individuelle Strukturen aufgrund unterschiedlicher Anordnung einzelner Funktionen innerhalb des Programms. Die Betriebssysteme der einzelnen Hersteller würden sich in verschiedenen Punkten wesentlich voneinander unterscheiden, je nachdem, welche Schwerpunkte ein Hersteller bei der Entwicklung setze; ob er z. B. mehr Wert auf Schnelligkeit, ein Arbeitsergebnis zu erzielen, oder aufi Bedienungskomfort lege, ob ein Betriebssystem die Erstellung von Anwendersoftware sowie deren Verarbeitung stärker - dann unter Umständen aufi Kosten der Geschwindigkeit - oder weniger stark unterstütze. Die Vielfalt der Variationsmöglichkeiten zeige sich darin,, daß es für zahlreiche Datenverarbeitungsanlagen jeweils unterschiedliche Betriebssysteme mehrerer 
Hersteller gebe, die also auf ein und derselben Hardware eingesetzt werden können; dies treffe aufi ihre Anlagen allerdings nur ausnahmsweise zu (z.B. aufidie Anlagen der Serie 8890). Sie kommt im übrigen auch in der als Anlage 2 zum Schriftsatz vom 2. März 1988 vorgelegten 111-seitigen Darstellung der Systemsoftware (einschließlich der Ausarbeitung „Fernbetreuung") sowie der vorgelegten Systemliteratur zum Ausdruck. Dort wird näher dargelegt, daß bei den einzelnen Programmen in der Auswahl, Sammlung und Anordnung der Informationen sowie in den gewählten Aufbau- und Einteilungsprinzipien eigenschöpferische Züge hervorträten (vgl. nachfolg. unter II. 3. b bb).

bb) Entgegen der Annahme des Berufungsgerichts ist das Klagevorbringen auch nicht deshalb unsubstantiiert, weil es „an einer der sinnlichen Wahrnehmung zugänglichen Synopse der konkreten und vorbekannten Formgestaltungen in den hier in Frage stehenden Phasen" fehle. Das Berufungsgericht hat auch insoweit zu hohe Anforderungen gestellt. Der zur Feststellung schöpferischer Eigenheiten gebotene Gesamtvergleich mit dem Vorbekannten setzt voraus, daß der Klägerin die Systemsoftware und die Arbeitsergebnisse der einzelnen Entwicklungsstufen anderer Hersteller mit ihren jeweils üblichen Anordnungen, Systemen, Aufbau- und Einteilungsprinzipien auch im einzelnen bekannt sind. Dazu wird aber in der Regel die Kenntnis der Quellprogramme anderer Hersteller erforderlich sein, die gerade hinsichtlich der Systemsoftware nicht ohne weiteres vorausgesetzt werden kann, wobei einschränkend noch hinzutritt, daß für die Datenverarbeitungsanlagen, auf: die sich die streitgegenständliche Systemsoftware bezieht, ohnehin keine Betriebssysteme anderer Hersteller angeboten werden. Verteidigt sich der Beklagte mit dem Einwand, die Schutzfähigkeit entfalle oder der Schutzumfang sei eingeschränkt, weil der Urheber aufi vorbekanntes Formengut zurückgegriffen habe, so ist es grundsätzlich seine Sache, die Gestaltung des Vorbekannten darzulegen und zu beweisen (vgl. BGH, Urt. v. 27. 5. 1981 - I ZR 102/79, GRUR 1981, 820, 822 - Stahlrohrstuhl II).

Die Revision weist im übrigen zu Recht darauf hin, daß die Klägerin an zahlreichen Stellen der Ausarbeitung gemäß Anlage 2 die Neuheit bestimmter Formgebungen, also deren individuelle Gestaltung durch sie, zum Ausdruck gebracht hat. So hat sie u. a. bei der Beschreibung des Systems TACOS (Transaction Control System), das den gleichzeitigen Ablauf bis zu 127 Anwenderprogramme ermöglichen soll, ausgeführt, daß es sich um ein einzigartiges Programmsystem handele, weil keiner der bis dahin bekannten - Wege zur Realisierung eines Dialogsystems beschritten worden sei; kein vergleichbares System aus dem Bereich der Batchverarbeitung sei durch ein vergleichbares Betriebssystem in ein ähnlich anwenderfreundliches Dialogsystem verwandelt worden (S. 3); zu der mit „Druck Spooling“ bezeichneten Aufgabe heißt es, auf einem Rechner der in Rede stehenden Kategorie sei ein solches Programmelement, mit dieser Konsequenz ausgeführt, einzigartig gewesen (S. 6); zum „Input Recovery“ wird aufi den Gegensatz zu herkömmlichen Systemen hingewiesen (S. 7). Im Kapitel „Magnetplatten, Terminals“ wird dargelegt, daß durch eine grundlegende Änderung im Betriebssystem besondere Zugriffsmöglichkeiten gegenüber vergleichbaren Systemen geschaffen worden seien (S. 26). Im Kapitel „Betriebssystem Eigenschaften“ wird die dynamische Textverwaltung hervorgehoben, die eine absolut neue Flexibilität zulasse, was Kundenwünsche und Kundenanforderungen anbelange (S. 28). Das Thema „Menüsteuerung TAMOS“ wird mit dem Hinweis aufi die Umstellung von einer kommandoorientierten Bedienersteuerung (IRIS) zu einer menüorientierten Benutzerführung (NIROS) eingeleitet und vorgetragen, daß das System der Klägerin mit dieser Eigenschaft zu seiner Zeit in dieser Rechnerklasse einzigartig gewesen sei (S. 30). Im Rahmen des Kapitels „Konfigurierung (SYSMOD)“ wird dargelegt, daß die ungewöhnliche Möglichkeit, ein Betriebssystem durch Parametrisierung neuen Hard- und Softwaregegebenheiten anpassen zu können, zur damaligen Zeit einzigartig und einer weit überdurchschnittlichen Programmiererleistung zu verdanken gewesen sei (S. 33). Unter „TKD Support, Fernbetreuung“ heißt es, daß bei Schaffung einer Service- und Testumgebung des Betriebssystems NIROS insbesondere die vielfältigen Abhängigkeiten unterschiedlicher Systemfunktionen zu berück-sichtigen gewesen seien, für die es wegen der Komplexität kein vorgegebenes Schema gegeben habe; es sei eine Fernbetreuungssoftware geschaffen worden, die bis heute auf: vergleichbaren Computersystemen anderer Hersteller nicht verfügbar sei (S. 37). Bei der Beschreibung des COBOL-Programmsystems wird schließlich hervorgehoben, daß ein Konzept der diagrammgesteuerten Syntaxanalyse mit Hilfe einer selbstentwickelten Programmsprache nebst dazugehörenden Umsetzungsprogrammen geschaffen worden

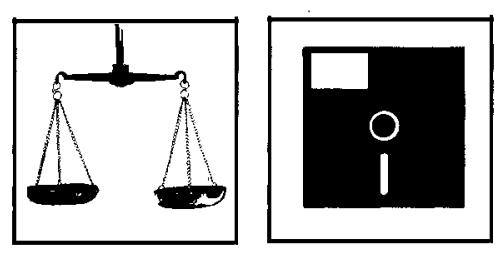

111 Seiten Darstellung der Systemsoftware als Anlage zum Schriftsatz

Das Problem des Vergleichs mit. dem Vorbekannten Der Wechselider Darlegungslast.
zum Beklagten bei Bestreiten.

\section{Beispieleffür individuelle Formgebungen}

„Transaction Control System“

„Druck Spooling“"

"Input Recovery"

Dynamische Textverwaltung:

Menüorientierte Benutzeffübrung.

Anpassung durch

Parametrisierung

Fernbetreuungssoftware

Diagrammgesteuerte Syptaxanahyse 1. 


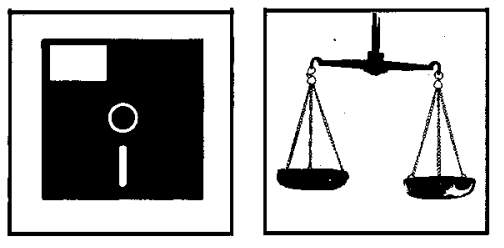

Abreichendivom OLG Hamm: Gestaltungshöhe wurde substantiiert beansprucht

Kein Anlaß zum Abrücken von der "Inkasso-Programm"Entscheidung

Gemeintwar:

Die allgemeinen Grundsätze des Urheberrechts'gelten auchifür Softzware

Gestaltungshöhe:

Von der Klägerin substantiiert dargelegt

Auf die Mannjabre kommt es (urbeberrechtlich) nicht an.
Urheberrechtsschutz für Computerprogramme

sei, für die es kein Vorbild gebe (S. 50). Diese Beispiele machen deutlich, daß die Klägerin zahlreiche schöpferische Eigenheiten gegenüber vorbekannten Formgestaltungen substantiiert aufgeführt hat.

cc) Abweichend vom Berufungsgericht ist auch davon auszugehen, daß die Klägerin eine für die Urheberrechtsschutzfähigkeit hinreichende Gestaltungshöhe ihrer Systemsoftware substantiiert beansprucht hat. Das Berufungsgericht hat offengelassen, ob die in BGHZ 94, 276 ff: aufgestellten Anforderungen im Blick auf: spätere Senatsentscheidungen niedriger anzusetzen seien. Es hat ausgeführt, daß im Klagevorbringen nirgends deutlich werde, wo in der Auswahl, Sammlung, Anordnung und Einteilung der Informationen schöpferische Eigenheiten liegen könnten. Die von der Klägerin für eigentümlich gehaltenen Leistungen seien nur kursorisch umschrieben, ohne daß sich daraus entnehmen ließe, ob die gewählten Strukturen technisch bedingt seien oder auf gestalterischen Elementen beruhten. Über „theoretische Umschreibungen“ und „Abstraktionen“ hinausgehende sinnlich wahrnehmbare Formgestaltungen seien nicht erkennbar.

Der Senat sieht keine Veranlassung - etwa im Blick aufi die vom Berufungsgericht angeführten späteren BGH-Entscheidungen -, von den Grundsätzen der Inkasso-ProgrammEntscheidung abzuweichen. In den angeführten Entscheidungen (BGH, Urt. v. 20. 11. 1986 - I ZR 160/84, GRUR 1987, 360, 361 - Werbepläne; Urt. v. 2. 7.1987 - I ZR 232/85, GRUR 1988, 33, 35 - Topographische Landeskarten) ist mit Rücksicht auf die Art der in Frage stehenden Werke als Darstellungen wissenschaftlicher und technischer Art und des für diese Werke eng begrenzten Schutzumfangs der für die Urheberrechtsschutzfähigkeit vorausgesetzte schöpferische Eigentümlichkeitsgrad nicht $\mathrm{zu}$ hoch angesetzt worden. Die Zweckbestimmung dieser - von $\S 2$ Abs. 1 Nr. 7 gleichwohl als schutzwürdig angesehenen - Darstellungen, wie Zeichnungen, Pläne, Skizzen, läßt für eine individuelle Gestaltung wenig Raum, so daß einerseits die Anforderungen an die Schutzfähigkeit nicht zu hoch gestellt und andererseits der Schutzumfang entsprechend eng begrenzt werden muß. Bei Datenverarbeitungsprogrammen bestehen dagegen - wie in der Inkasso-Programm-Entscheidung (BGHZ 94, 276, 285 f) dargelegt - vielfältige Möglichkeiten einer individuellen schöpferischen Gestaltung. Dementsprechend sind die Anforderungen bei ihnen nicht zu niedrig anzusetzen; die Gestaltung muß jedenfalls das handwerkliche Durchschnittskönnen erheblich überragen. Diese an der üblichen urheberrechtlichen Diktion ausgerichtete Formulierung enthält keine gegenüber den allgemeinen urheberrechtlichen Grundsätzen verschärften Anforderungen für Datenverarbeitungsprogramme, sondern überträgt diese Grundsätze aufi und nach Maßgabe dieser besonderen Werkart (vgl. aus der Rechtsprechung zum Schriftwerkschutz u.a. BGH, Urt. v. 29. 3. 1984 - I ZR 32/82, GRUR 1984, 659, 661 - Ausschreibungsunterlagen; Urt. v. 17. 4. 1986 - I ZR 213/83, GRUR 1986, 739, 741 - Anwaltsschriftsatz; Urt. v. 12. 3. 1987 - I ZR 71/85, GRUR 1987, 704, 706 - Warenzeichenlexika).

Die Klägerin hat hier im einzelnen hinreichend substantiiert dargelegt und unter Beweis gestellt, daß ihre Systemsoftware die danach erforderliche Gestaltungshöhe erreicht. Vergleichsmaßstab für die Ermittlung der nötigen Gestaltungshöhe ist im konkreten Einzelfall das alltägliche, durchschnittliche Schaffen bei der Programmerstellung, das aufe einer mehr oder weniger routinemäßigen, handwerksmäßigen, mechanisch-technischen Aneinanderreihung und Zusammenfügung des Materials beruht. Dagegen wäre es verfehlt, dem Urheber der in Frage stehenden Formgestaltung einen durchschnittlichen oder „ähnlich guten“ Gestalter als Vergleichsperson gegenüberzustellen (vgl. BGH, Urt. v. 17. 4. 1986 - I ZR 213/83, GRUR 1986, 739, 741 - Anwaltsschriftsatz).

Die Klägerin hat substantiiert vorgetragen, daß die streitgegenständliche Systemsoftware das durchschnittliche Schaffen auf diesem Gebiet deutlich übersteigt und nichts mit alltäglicher Routine zu tun hat. Dies folgt zwar nicht entscheidend aus ihrem - eher den wettbewerblichen Besitzstand dokumentierenden - Vorbringen, daß der personelle Aufwand für die Erstellung eines einfachen Betriebssystems zwischen 100 und 200 Mannjahre betrage, daß allein das NIROS-Betriebssystem aus ca. 1,5 Mio. Programmschritten bestehe und daß der finanzielle Entwicklungsaufwand der Betriebssysteme NIROS und TAMOS sich auf: ein Mehrfaches eines zweistelligen Millionenbetrages belaufe. Denn für die Frage der schöpferischen Gestaltungshöhe kommt es grundsätzlich weder auf den quantitativen Umfang noch daraufi an, mit welchem Aufwand und mit welchen Kosten ein Programm konzipiert worden ist (vgl. BGHZ 94, 276, 287 - InkassoProgramm). Gleichwohl können diese Umstände auch für die Schaffung einer außeror- 
dentlich komplexen Systemsoftware sprechen, die nicht aufi einer bloß mechanischen, aufgrund allgemein bekannter Anweisungen durchgeführten Tätigkeit beruht, sondern wie von der Klägerin im einzelnen ausgeführt - auch erhebliche Anforderungen an die schöpferische Leistung der Programmautoren stellt.

Die Klägerin hat sich hierzu im einzelnen aufi ihre Anlage 2 zum Schriftsatz vom 2. März 1988 sowie die beigefügte Systemliteratur und weiter aufi das von ihr vorgelegte Privatgutachten Profi Lewandowski vom 13. April 1986 berufen, der ausgeführt hat, daß Betriebssysteme für mittlere und größere Datenverarbeitungsanlagen in der Regel einen sehr hohen Grad an Individualität erreichen; die Implementierung stelle sowohl an die Entwickler der Betriebssystem-Konzeption als auch an die Realisierer der Konzepte erhebliche Anforderungen, die über die Möglichkeiten eines durchschnittlichen Programmierers weit hinausgingen (S. 5). Die Klägerin hat überdies auf das Gutachten des vom Landgericht bestellten Sachverständigen Lautz verwiesen, soweit darin dargelegt wird, daß Steuerprogramme stets komplizierte Programme seien, daß Programmierarbeiten am Betriebssystem sehr aufwendig seien, weil sie sehr gründlich geplant und durchgeführt werden müßten, und daß die Programmierung nicht nur große Konzentration, sondern auch schöpferische Phantasie erfordere. Letztlich hat die Klägerin auch Meinungen im Schrifttum angeführt, die Betriebssysteme als Programme hohen Schwierigkeitsgrads beurteilen, bei denen sich eine persönliche geistige Schöpfung in der Auswahl, Einteilung und Anordnung der Informationen in besonders hohem Maße zeige (Kindermann, CR 1986, 446; vgl. auch v. Gravenreuth, BB 1985, 2002, 2003).

c) Unter diesen Umständen hätte das Berufungsgericht das durch zahlreiche Meinungsäußerungen gestützte Vorbringen der Klägerin zur Urheberrechtsschutzfähigkeit von Systemsoftware der streitgegenständlichen Art nicht als unsubstantiiert behandeln dürfen, zumal es vorliegend nicht aufi eine genaue Festlegung der eigenschöpferischen Elemente im einzelnen ankommt (vgl. oben II. 3. a), sondern die Feststellung ausreicht, daß die Systemsoftware überhaupt solche Elemente aufweist.

Die Sache ist daher zur weiteren tatrichterlichen Aufklärung an das Berufungsgericht zurückzuverweisen. Das Berufungsgericht wird das von der Klägerin beantragte Sachverständigengutachten einzuholen haben, um zu klären, ob sich in der Sammlung, Auswahl und Gliederung der Befehle und einer Vielzahl variierbarer Zweckmäßigkeitserwägungen eigenschöpferische Züge zeigen. Es wird dabei vor allem aufidie individuelle (formale) Programmstrukturen abzustellen haben mit der häufigen Benutzung von Grundfunktionen für eine Vielzahl von unterschiedlichen Anwendungsprogrammen, der Art, wie Unterprogramme, Arbeitsroutinen, mit Verzweigungsanweisungen verknüpft werden u.ä.; dazu gehört auch die Verwendung von Algorithmen, die zwar als solche einem Urheberrechtsschutz nicht zugänglich sind (BGHZ 94, 276, 285 - InkassoProgramm), wohl aber in der Art und Weise der Implementierung und Zuordnung zueinander urheberrechtsschutzfähig sein können (vgl. OLG Frankfurt GRUR 1985, 1049, 1050; dazu Nichtannahmebeschluß des BGH v. 26. 9. 1985 - I ZR 219/84). Nicht die Rechenregel, die Idee, die mathematische Formel ist hier Gegenstand des Schutzes, sondern das "Gewebe" (vgl. E. Ulmer, Der Urheberrechtsschutz wissenschaftlicher Werke unter besonderer Berücksichtigung der Programme elektronischer Rechenanlagen, 1967; S. 3).

d) Die weitere Aufklärung wäre nur dann entbehrlich, wenn die Klage selbst bei bestehendem Urheberrechtsschutz - wie vom Landgericht angenommen - unter dem Gesichtspunkt der Erschöpfung des Verbreitungsrechts ( $§ 17$ Abs. 2 UrhG) abzuweisen wäre. Diese vom Berufungsgericht - von seinem Standpunkt aus folgerichtig - bislang offengelassene Frage läßt sich ohne weitere tatrichterliche Feststellungen noch nicht abschließend entscheiden. Denn auch bei unterstellter Erschöpfung würde sich diese nur aufi eine Weiterverbreitung der konkreten Systemsoftware an Dritte und nicht aufi die von den Klageanträgen ebenfalls erfaßte Veränderung und Vervielfältigung beziehen. Im übrigen könnte auch im Falle der Weiterveräußerung geänderter Systemsoftware grundsätzlich keine Erschöpfung eintreten. Selbst wenn entgegen der Ansicht der Klägerin die Nutzungsüberlassung als - wie für den Eintritt der Erschöpfung nach $\S 17$ Abs. 2 UrhG erforderlich - Veräußerung zu werten wäre (vgl. dazu neuerdings LG Nürnberg-Fürth CR 1990, 118 ff), wäre daher noch klärungsbedürftig, ob die Beklagten - wie die Klägerin behauptet - urheberrechtlich relevante Veränderungen am Betriebssystem vorgenommen haben.

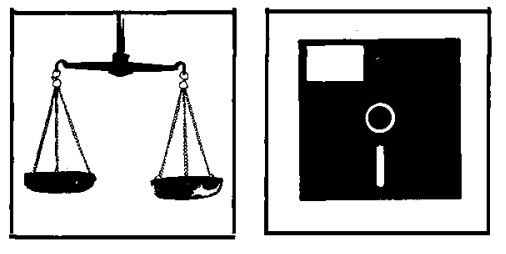

Das von Nixdorf vorgelegte

Privatgutachten

Das Gutachten des vom $L G$

Bielefeld, bestellien

Sachverständigen

Es reicht aus, daß überhaupt eigenschöpferische Elemente dar'gelegt sind.

Zurückverweisung mit der Auflage, das beantragte Sachverständigengutachten einzubolen

Geschützt ist das „Gezeebe“

Erschöpfung des Verbreitungsrechts nach $\$ 17$ Abs. 2 UrbG?

Vervielfältigung bei SoftwerareNutzung? 

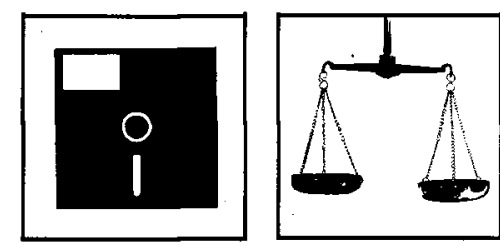

Vervielfältigung, bei Abspeicherung und.Ausdruck

Keine Vervielfältigung durch Aus:gabe auf dem Bildschirm

Die Benutzung, , als solche“ ist nie
urheberrechtlich relevant

Auch wettbewerbsrechtliche Ansprüche bleiben zuiprüfen. Ansprüche aus wettbewerbsrechtlichem Leistungsschutz?

Ausnutzen fremden Vertragsbruchs?

Sollte es bei der weiteren Prüfung aufi die Frage einer unzulässigen Vervielfältigung ankommen, wird das Berufungsgericht zu beachten haben, daß bei der Benutzung von Computerprogrammen rein technisch verschiedene tatsächliche Vervielfältigungsvorgänge anfallen können, die nicht uneingeschränkt vom Vervielfältigungsrecht nach § 16 UrhG erfaßt werden. Im Schrifttum besteht weitgehende Übereinstimmung darin, daß jedenfalls in der Abspeicherung des Programms aufi einen Datenträger (Festplatte, Diskette u.ä.) und im Ausdruck des Programms in seiner Konfiguration mittels eines Druckers eine Vervielfältigung im Sinne des §16 UrhG zu sehen ist (vgl. Schricker/ Loewenheim, Urheberrecht, 1987, § 16 Rdn. 9 m.w.N.). Diese Vorgänge fallen unter den Vervielfältigungsbegriff; der jede körperliche Festlegung eines Werkes erfaßt, die geeignet ist, das Werk den menschlichen Sinnen aufi irgendeine Weise unmittelbar oder mittelbar wahrnehmbar zu machen (vgl. Amtl. Begr., BT-Drucks. IV/270 S. 47; BGH, Urt. v. 3. 7. 1981 - I ZR 106/79, GRUR 1982, 102, 103 - Masterbänder; BGH, Urt. v. 1. 7. 1982 - I ZR 119/80, GRUR 1983, 28, 29 - Presseberichterstattung und Kunstwerkwiedergabe II). Umstritten ist, ob auch beim reinen Ablaufi des Programms im Computer, bei der eigentlichen Programmbenutzung, Vervielfältigungsvorgänge in diesem Sinne anfallen. Dabei scheidet die Ausgabe des Programms aufi dem Bildschirm von vorneherein als maßgeblicher Vervielfältigungstatbestand aus, da insoweit nur eine unkörperliche Wiedergabe vorliegt (vgl. u.a. E. Ulmer, GRUR 1971, 297, 301; Katzenberger, GRUR 1973, 629, 632; Schricker/Loewenheim aaO $\S 16 \mathrm{Rdn}$. 9). Im übrigen ist zu beachten, daß die reine Benutzung - im Gegensatz zu den technischen Nutzungsrechten - urheberrechtlich nicht erfaßt wird. Die Benutzung eines Werkes als solche ist kein urheberrechtlich relevanter Vorgang. Dies gilt für das Benutzen eines Computerprogramms ebenso wie für das Lesen eines Buches, das Anhören einer Schallplatte, das Betrachten eines. Kunstwerks oder eines Videofilms. Es wird daher auf die Frage ankommen, ob die im Rahmen der Programmbenutzung erfolgende Programmeingabe und -verarbeitung eine Vervielfältigung erforderlich macht.

4. Auch soweit das Berufungsgericht wettbewerbsrechtliche Ansprüche verneint hat, halten seine Ausführungen der rechtlichen Nachprüfung nicht stand.

$\mathrm{Ob}$ der Klägerin Ansprüche unter dem Gesichtspunkt des wettbewerbsrechtlichen Leistungsschutzes zustehen, hängt im Blick aufi die zu fordernde wettbewerbliche Eigenart letztlich ebenfalls von den weiteren Feststellungen zur Urheberrechtsschutzfähigkeit ab. Das Berufungsgericht wird allerdings zu beachten haben, daß es bereits am Nachahmungstatbestand fehlt, sofern die Beklagten die mit der angekauften Hardware erworbene Systemsoftware lediglich weiterveräußert haben, ohne die fremde Leistung selbständig zu übernehmen.

Die Revision rügt weiter zu Recht, daß das Berufungsgericht den Handel der Beklagten mit gebrauchten Computern einschließlich der Systemsoftware der Klägerin nicht unter dem Gesichtspunkt der Ausnutzung fremden Vertragsbruchs als selbständige Verletzungshandlung geprüft hat. Auch insoweit sind weitere Feststellungen erforderlich; denn das Ausnutzen fremden Vertragsbruchs ist nicht als solches, sondern erst bei Hinzutreten weiterer Unlauterkeitsmerkmale wettbewerbswidrig.

III.

Das Berufungsurteil war daher aufzuheben und die Sache zur anderweiten Verhandlung und Entscheidung, auch über die Kosten der Revision, an das Berufungsgericht zurückzuverweisen.
v. Gamm
Erdmann
Teplitzky
Mees Ullmann 